\title{
Evolutionary $p$-Laplacian with convection and reaction under dynamic boundary condition
}

\author{
Shanming $\mathrm{Ji}^{1}$, Jingxue Yin ${ }^{1}$ and Rui Huang ${ }^{1,2^{*}}$
}

\section{"Correspondence:}

huangrui@m.scnu.edu.cn

'School of Mathematical Sciences,

South China Normal University,

Guangzhou, 510631, China

${ }^{2}$ Department of Mathematics,

South China University of

Technology, Guangzhou, 510640,

China

\begin{abstract}
We study the global existence and blow-up phenomenon of solutions to an evolutionary $p$-Laplacian with convection and reaction under dynamic boundary condition.
\end{abstract}

Keywords: dynamic boundary condition; blow up; $p$-Laplacian; reaction-diffusion

\section{Introduction}

In this paper, we are concerned with the following evolutionary $p$-Laplacian under dynamic boundary condition:

$$
\begin{aligned}
& \frac{\partial u}{\partial t}=\operatorname{div}\left(|\nabla u|^{p-2} \nabla u\right)-\vec{g}(u) \cdot \nabla u+f(u), \quad x \in \Omega, t>0, \\
& \sigma u_{t}+|\nabla u|^{p-2} \nabla u \cdot v=0, \quad x \in \partial \Omega, t>0, \\
& u(x, 0)=u_{0}(x), \quad x \in \Omega,
\end{aligned}
$$

where $p>1, \vec{g}: \mathbb{R} \rightarrow \mathbb{R}^{N}, f: \mathbb{R} \rightarrow \mathbb{R}, \Omega \subset \mathbb{R}^{N}$ is a bounded domain with smooth boundary $\partial \Omega$, and $v: \partial \Omega \rightarrow \mathbb{R}^{N}$ is the outer unit normal vector.

The quasilinear parabolic problems with dynamic boundary conditions of type (1.1)(1.3) arise in numerous areas such as heat conduction, chemical reactor theory, colloid chemistry and population growth, see $[1,2]$ and the references therein. Many reactiondiffusion equations under dynamic boundary conditions have been considered in the past years. An early study of problem (1.1)-(1.3) with $p=2$ and $\vec{g}=\overrightarrow{0}$ was carried out by Below and Mailly [3] who showed a complete result about the blow-up phenomena as well as the lower and upper bounds for the blow-up time. Moreover, some of the techniques were also applied to the porous medium equation with reaction. Later on, for the evolutionary $p$ Laplacian with $p \geq 2 N /(N+2)$, where $N$ is the dimension of the domain, Gal and Warma [4] considered the following equation without convection:

$$
\frac{\partial u}{\partial t}-\operatorname{div}\left(|\nabla u|^{p-2} \nabla u\right)+f(u)=g(x), \quad x \in \Omega, t>0,
$$

(c) $2015 \mathrm{Ji}$ et al. This article is distributed under the terms of the Creative Commons Attribution 4.0 International License (http://creativecommons.org/licenses/by/4.0/), which permits unrestricted use, distribution, and reproduction in any medium, provided you give appropriate credit to the original author(s) and the source, provide a link to the Creative Commons license, and indicate if changes were made. 
coupled with dynamic boundary conditions. The well-posedness and the existence of a global attractor results were established. More recently, Mailly and Rault [2] studied the nonlinear convection problem (1.1)-(1.3) with $p=2$ and proved the global existence and blow-up phenomena of local solutions. For other results about the solvability of quasilinear parabolic equations with dynamic boundary conditions, we refer the readers to [5-7], etc.

Throughout this paper, we suppose that the dissipativity condition holds

$$
\sigma>0, \quad \sigma \in C^{1}(\partial \Omega \times[0,+\infty))
$$

and the functions in problem (1.1)-(1.3) are smooth

$$
f \in C^{1}(\mathbb{R}), \quad f(s) \geq 0 \quad \text { for } s \geq 0, \quad \vec{g} \in C^{1}\left(\mathbb{R}, \mathbb{R}^{N}\right),
$$

the initial data is non-negative and satisfies

$$
u_{0} \geq 0, \quad u_{0} \in L^{\infty}(\Omega) \cap W^{1, p}(\Omega) .
$$

In Section 2 we develop the comparison principle for a regularized problem and the local existence of weak and strong solutions of problem (1.1)-(1.3). In Section 3 we derive the global existence of the strong solutions, while in Section 4 we prove the blow-up phenomenon of strong solutions by formulating a family of radially symmetric lower solutions.

\section{Comparison principle and local existence}

In this section, we use the regularization method and compactness theorems to prove the local existence of the solutions to problem (1.1)-(1.3).

Consider the following regularized problem:

$$
\begin{aligned}
& \frac{\partial u}{\partial t}=\operatorname{div}\left(\left(\frac{1}{n}+|\nabla u|^{2}\right)^{\frac{p-2}{2}} \nabla u\right)-\vec{g}\left(u_{+}\right) \cdot \nabla u+f_{M}(u), \quad x \in \Omega, t>0, \\
& \sigma u_{t}+\left(\frac{1}{n}+|\nabla u|^{2}\right)^{\frac{p-2}{2}} \nabla u \cdot v=0, \quad x \in \partial \Omega, t>0, \\
& u(x, 0)=u_{0, n}(x), \quad x \in \Omega,
\end{aligned}
$$

where $f_{M}(s)=\min \left\{f_{+}(s), M\right\}, s_{+}=\max \{s, 0\}, M>0, n \in \mathbb{Z}^{+}, u_{0, n} \in C^{\infty}(\bar{\Omega})$ satisfies

$$
\inf _{\Omega} u_{0} \leq u_{0, n} \leq \sup _{\Omega} u_{0}, \quad\left\|u_{0, n}\right\|_{W^{1, p}} \leq 2\left\|u_{0}\right\|_{W^{1, p}}, \quad \lim _{n \rightarrow \infty}\left\|u_{0, n}-u_{0}\right\|_{W^{1, p}}=0 .
$$

Since $f, \vec{g} \in C^{1}$, we can verify that $f_{M}, \vec{g}\left(s_{+}\right)$are locally Lipschitz continuous.

Hereafter, we suppose that the regularized problem (2.1)-(2.3) has a classical solution $u_{n, M} \in C^{2,1}\left(\bar{\Omega} \times\left[0, T_{n, M}\right)\right)$ with the maximal existence time $0<T_{n, M} \leq+\infty$. Let $Q_{T}=\bar{\Omega} \times$ 
$(0, T)$ for $T>0$ and define

$$
\begin{aligned}
& F_{n, M}[u]=F_{n, M}(u, \nabla u)=\operatorname{div}\left(\left(\frac{1}{n}+|\nabla u|^{2}\right)^{\frac{p-2}{2}} \nabla u\right)-\vec{g}\left(u_{+}\right) \cdot \nabla u+f_{M}(u), \\
& B_{n}[u]=\sigma u_{t}+\left(\frac{1}{n}+|\nabla u|^{2}\right)^{\frac{p-2}{2}} \nabla u \cdot v .
\end{aligned}
$$

Notice that, in the dynamic boundary condition, $B_{n}[u]$ is nonlinear with respect to $\nabla u$. First, we need the following comparison principles which are simple variations of the comparison principles in [8].

Lemma 2.1 Let $u, v \in C^{2,1}\left(Q_{T}\right) \cap C\left(\bar{Q}_{T}\right)$ satisfying

$$
\begin{aligned}
& u_{t}-F_{n, M}[u]>v_{t}-F_{n, M}[v], \quad(x, t) \in Q_{T}, \\
& B_{n}[u]>B_{n}[v], \quad(x, t) \in \partial \Omega \times(0, T), \\
& u(x, 0)>v(x, 0), \quad x \in \bar{\Omega} .
\end{aligned}
$$

Then

$$
u(x, t)>v(x, t), \quad(x, t) \in Q_{T} .
$$

Proof Suppose that there exists $\left(x_{0}, t_{0}\right) \in Q_{T}$ such that $u\left(x_{0}, t_{0}\right) \leq v\left(x_{0}, t_{0}\right)$. Let

$$
t^{*}=\sup \left\{\tau \in(0, T) ; u(x, t)>v(x, t), \forall(x, t) \in Q_{\tau}\right\} .
$$

Then $t^{*} \in\left(0, t_{0}\right] \subset(0, T)$ and $\min _{\bar{Q}_{t^{*}}}\{u-v\}=0$. Thus, $u-v$ attains its minimum 0 at some point $\left(x^{*}, t^{*}\right)$ with $x^{*} \in \bar{\Omega}$. If $x^{*} \in \Omega$, then

$$
u=v, \quad u_{t} \leq v_{t}, \quad \nabla u=\nabla v, \quad D^{2} u \geq D^{2} v \quad \text { at }\left(x^{*}, t^{*}\right),
$$

which contradicts $u_{t}-F_{n, M}[u]>v_{t}-F_{n, M}[v]$. If $x^{*} \in \partial \Omega$, then

$$
u_{t} \leq v_{t}, \quad \frac{\partial u}{\partial v} \leq \frac{\partial v}{\partial v}, \quad \frac{\partial u}{\partial \mu_{i}}=\frac{\partial v}{\partial \mu_{i}} \quad \text { at }\left(x^{*}, t^{*}\right)
$$

where $\frac{\partial}{\partial \mu_{i}}, i=1,2, \ldots, N-1$, are the tangential derivatives in the local coordinates at $\left(x^{*}, t^{*}\right)$. We can verify that

$$
\left(\frac{1}{n}+|\nabla u|^{2}\right)^{\frac{p-2}{2}} \nabla u \cdot v=\left(\frac{1}{n}+\sum_{i=1}^{N-1}\left|\frac{\partial u}{\partial \mu_{i}}\right|^{2}+\left|\frac{\partial u}{\partial v}\right|^{2}\right)^{\frac{p-2}{2}} \frac{\partial u}{\partial v}
$$

which is increasing with respect to $\frac{\partial u}{\partial v}$ since $p>1$. Therefore, $B_{n}[u] \leq B_{n}[v]$. We arrive at another contradiction.

Using Lemma 2.1, we can prove the following comparison principle, which is similar to Theorem 2.2 in [8], but without the global one-side Lipschitz condition. 
Lemma 2.2 Let $u, v \in C^{2,1}\left(Q_{T}\right) \cap C\left(\bar{Q}_{T}\right)$ satisfying

$$
\begin{aligned}
& u_{t}-F_{n, M}[u] \geq v_{t}-F_{n, M}[v], \quad(x, t) \in Q_{T}, \\
& B_{n}[u] \geq B_{n}[v], \quad(x, t) \in \partial \Omega \times(0, T), \\
& u(x, 0) \geq v(x, 0), \quad x \in \bar{\Omega} .
\end{aligned}
$$

Then

$$
u(x, t) \geq v(x, t), \quad(x, t) \in Q_{T} .
$$

Proof For any given $T>0, \varepsilon>0$, since $u, v \in C(\bar{\Omega} \times[0, T])$, by the continuities and $u(x, 0) \geq$ $v(x, 0)$, there exists $\delta=\delta_{\varepsilon}>0$ such that

$$
u(x, t)>v(x, t)-\varepsilon, \quad x \in \bar{\Omega}, t \in(0, \delta]
$$

Notice that $v \in C^{2,1}(\bar{\Omega} \times[\delta, T-\varepsilon]), v_{+}(x, t) \in\left[0, \max _{\bar{Q}_{T}} v\right]$, and $\vec{g} \in C^{1}\left(\left[0, \max _{\bar{Q}_{T}} v\right]\right)$. There exists a constant $K>0$ such that

$$
\begin{aligned}
& \left|\vec{g}\left((v-s)_{+}\right) \cdot \nabla v-\vec{g}\left(v_{+}\right) \cdot \nabla v\right| \leq \sup _{\bar{\Omega} \times[\delta, T-\varepsilon]}|\nabla v| \cdot\left|\vec{g}\left((v-s)_{+}\right)-\vec{g}\left(v_{+}\right)\right| \leq \frac{K}{2} s, \\
& \left|f_{M}(v-s)-f_{M}(v)\right| \leq \frac{K}{2} s, \quad s \geq 0 .
\end{aligned}
$$

Define $\varphi=v-\varepsilon e^{(K+1)(t-\delta)}$. Thus,

$$
\begin{aligned}
& \varphi_{t}-F_{n, M}[\varphi] \leq v_{t}-(K+1) \varepsilon e^{(K+1)(t-\delta)}-F_{n, M}[v]+K \varepsilon e^{(K+1)(t-\delta)} \\
& \quad<v_{t}-F_{n, M}[v] \leq u_{t}-F_{n, M}[u], \quad(x, t) \in \Omega \times[\delta, T-\varepsilon], \\
& B_{n}[\varphi]=B_{n}[v]-(K+1) \sigma \varepsilon e^{(K+1)(t-\delta)}<B_{n}[v] \leq B_{n}[u], \quad(x, t) \in \partial \Omega \times(\delta, T-\varepsilon), \\
& \varphi(x, \delta)=v(x, \delta)-\varepsilon<u(x, \delta), \quad x \in \bar{\Omega} .
\end{aligned}
$$

Lemma 2.1 implies $u(x, t) \geq \varphi(x, t)$ for $(x, t) \in \Omega \times\left[\delta_{\varepsilon}, T-\varepsilon\right]$. Therefore, $u(x, t) \geq$ $\min \left\{v(x, t)-\varepsilon, v(x, t)-\varepsilon e^{(K+1)\left(t-\delta_{\varepsilon}\right)}\right\}$ for $(x, t) \in \Omega \times(0, T-\varepsilon]$. By the arbitrariness of $\varepsilon>0$, we deduce $u(x, t) \geq v(x, t)$ for $(x, t) \in \Omega \times(0, T)$.

Lemma 2.3 There exists at most one classical solution of problem (2.1)-(2.3).

Proof Lemma 2.2 yields the uniqueness of classical solutions of problem (2.1)-(2.3).

Lemma 2.4 The solution $u_{n, M}$ of problem (2.1)-(2.3) satisfies

$$
\inf _{\Omega} u_{0} \leq u_{n, M}(x, t) \leq \sup _{\Omega} u_{0}+M t, \quad(x, t) \in \bar{\Omega} \times\left(0, T_{n, M}\right) .
$$

Thus, the maximal existence time $T_{n, M}=+\infty$. 
Proof For any given $T \in\left(0, T_{n, M}\right)$ and $\varepsilon>0$, define $\underline{u}_{\varepsilon}=\inf _{\Omega} u_{0}-\varepsilon-\varepsilon t, \bar{u}_{\varepsilon}=\sup _{\Omega} u_{0}+\varepsilon+$ $(M+\varepsilon) t$. Then

$$
\begin{aligned}
& \frac{\partial u_{n, M}}{\partial t}-F_{n, M}\left[u_{n, M}\right]=0>-\varepsilon \geq \frac{\partial \underline{u}_{\varepsilon}}{\partial t}-F_{n, M}\left[\underline{u}_{\varepsilon}\right], \\
& B_{n}\left[u_{n, M}\right]=0>-\sigma \varepsilon=B_{n}\left[\underline{u}_{\varepsilon}\right], \\
& u_{n, M}(x, 0)=u_{0, n}(x)>u_{0}(x)-\varepsilon \geq \underline{u}_{\varepsilon} .
\end{aligned}
$$

Lemma 2.1 implies $u_{n, M} \geq \underline{u}_{\varepsilon}$. Since $\varepsilon>0$ is arbitrary, we have $u_{n, M} \geq \inf _{\Omega} u_{0}$. The proof of $u_{n, M} \leq \sup _{\Omega} u_{0}+M t$ follows similarly.

Lemma 2.5 For $M_{1} \geq M_{2}>0$, there holds

$$
u_{n, M_{1}} \geq u_{n, M_{2}}, \quad x \in \Omega, t>0 .
$$

Proof For any given $T>0$, we see that $u_{n, M_{1}}, u_{n, M_{2}} \in C^{2,1}\left(Q_{T}\right) \cap C\left(\bar{Q}_{T}\right)$ and $f_{M_{1}}(s) \geq f_{M_{2}}(s)$ for $s \in \mathbb{R}$. Thus,

$$
\frac{\partial u_{n, M_{1}}}{\partial t}-F_{n, M_{2}}\left[u_{n, M_{1}}\right] \geq \frac{\partial u_{n, M_{1}}}{\partial t}-F_{n, M_{1}}\left[u_{n, M_{1}}\right]=0=\frac{\partial u_{n, M_{2}}}{\partial t}-F_{n, M_{2}}\left[u_{n, M_{2}}\right] .
$$

Using Lemma 2.2, we complete this proof.

Lemma 2.6 There exist constants $\delta_{0}, M_{0}>0$ independent of $n, M$ such that

$$
\sup _{n, M} u_{n, M}(x, t) \leq \sup _{\Omega} u_{0}+M_{0} \delta_{0}, \quad x \in \bar{\Omega}, t \in\left(0, \delta_{0}\right]
$$

Proof Let $\underline{u}_{0}=\inf _{\Omega} u_{0}$ and $\bar{u}_{0}=\sup _{\Omega} u_{0}$. Set $M_{0}=2 \max _{s \in\left\{\underline{u}_{0}, \bar{u}_{0}\right\}} f(s)+1$ and define

$$
h(t)=\max _{\underline{u}_{0} \leq s \leq \bar{u}_{0}+M_{0} t}|f(s)| .
$$

Since $f \in C^{1}\left(\left[\underline{u}_{0}, \bar{u}_{0}+M_{0}\right]\right)$, we see that $h$ is Lipschitz continuous on $[0,1]$ and $h(0)=$ $\max _{s \in\left\{\underline{u}_{0}, \bar{u}_{0}\right\}} f(s)<M_{0}$. Thus, there exists a constant $0<\delta_{0}<1$ such that $h(t)<M_{0}$ for all $t \in\left[0, \delta_{0}\right]$. By Lemma 2.4, $u_{n, M_{0}} \in\left[\underline{u}_{0}, \bar{u}_{0}+M_{0} t\right]$. Therefore,

$$
f\left(u_{n, M_{0}}(x, t)\right) \leq h(t)<M_{0}, \quad x \in \bar{\Omega}, t \in\left[0, \delta_{0}\right]
$$

and

$$
f_{M_{0}}\left(u_{n, M_{0}}(x, t)\right)=\min \left\{f\left(u_{n, M_{0}}(x, t)\right), M_{0}\right\}=f\left(u_{n, M_{0}}(x, t)\right), \quad(x, t) \in \bar{\Omega} \times\left(0, \delta_{0}\right] .
$$

If $M^{\prime} \leq M_{0}$, Lemma 2.5 implies

$$
u_{n, M^{\prime}} \leq u_{n, M_{0}} \leq \sup _{\Omega} u_{0}+M_{0} \delta_{0}, \quad(x, t) \in \bar{\Omega} \times\left(0, \delta_{0}\right]
$$

If $M^{\prime}>M_{0}$, since $u_{n, M^{\prime}} \in C\left(\bar{Q}_{\delta_{0}}\right)$ and $u_{n, M^{\prime}}(x, 0)=u_{0, n}(x) \in\left[\underline{u}_{0}, \bar{u}_{0}\right]$, we have

$$
f\left(u_{n, M^{\prime}}(x, 0)\right) \leq h(0)<M_{0}, \quad x \in \bar{\Omega},
$$


and there exists a constant $\delta_{M^{\prime}}>0$ such that

$$
f\left(u_{n, M^{\prime}}(x, t)\right)<M_{0}, \quad(x, t) \in \bar{\Omega} \times\left(0, \delta_{M^{\prime}}\right] .
$$

Thus,

$$
f_{M_{0}}\left(u_{n, M^{\prime}}(x, t)\right)=f\left(u_{n, M^{\prime}}(x, t)\right)=f_{M^{\prime}}\left(u_{n, M^{\prime}}(x, t)\right), \quad(x, t) \in \bar{\Omega} \times\left(0, \delta_{M^{\prime}}\right] .
$$

We see that $u_{n, M_{0}}, u_{n, M^{\prime}}$ are two classical solutions of problem (2.1)-(2.3) with $M=M_{0}$ on $\bar{\Omega} \times\left(0, \delta_{M^{\prime}}\right]$. According to the uniqueness, Lemma 2.3 , we have

$$
u_{n, M_{0}}(x, t)=u_{n, M^{\prime}}(x, t), \quad(x, t) \in \bar{\Omega} \times\left(0, \delta_{M^{\prime}}\right] .
$$

By the continuity of $u_{n, M^{\prime}}(x, t)$ and inequality (2.5), we can take $\delta_{M^{\prime}}=\delta_{0}$ in inequality (2.6). Then we have

$$
u_{n, M^{\prime}}(x, t)=u_{n, M_{0}}(x, t) \leq \sup _{\Omega} u_{0}+M_{0} \delta_{0}, \quad(x, t) \in \bar{\Omega} \times\left(0, \delta_{0}\right] .
$$

We arrive at a locally uniform bound of $u_{n, M}$.

Remark Lemma 2.4 shows that $u_{n, M} \leq \sup _{\Omega} u_{0}+M t$. However, the family $\left\{\sup _{\Omega} u_{0}+\right.$ $M t\}_{M>0}$ is not uniformly bounded on any interval $(0, \delta], \delta>0$. Lemma 2.6 provides the locally uniform bound of $u_{n, M}$.

Next, we derive some estimates on the solution $u_{n, M}$.

Lemma 2.7 Suppose that $\sigma$ does not depend on time. For any given $T>0, M>0$, there exists a constant $C=C(M, T)$ independent of $n$ such that

$$
\int_{\Omega} u_{n, M}^{2}(x, t) d x, \quad \int_{\partial \Omega} \sigma u_{n, M}^{2}(x, t) d S, \quad \int_{Q_{T}}\left|\nabla u_{n, M}\right|^{p} d x d t \leq C, \quad t \in(0, T) .
$$

Moreover, if $T=\delta_{0}$ (the constant in Lemma 2.6), then the constant $C=C\left(\delta_{0}\right)$ is independent of $n, M$.

Proof We write $u=u_{n, M}$ in this proof for the sake of convenience. Since $u \in C^{2,1}\left(Q_{T}\right) \cap$ $C\left(\bar{Q}_{T}\right)$, multiplying equation (2.1) by $u$ and integrating by parts over $Q_{\tau}, \tau \in(0, T]$, we have

$$
\begin{gathered}
\int_{Q_{\tau}} u u_{t} d x d t+\int_{Q_{\tau}}\left(\frac{1}{n}+|\nabla u|^{2}\right)^{\frac{p-2}{2}}|\nabla u|^{2} d x d t \\
-\int_{0}^{\tau} \int_{\partial \Omega} u\left(\frac{1}{n}+|\nabla u|^{2}\right)^{\frac{p-2}{2}} \nabla u \cdot v d S d t \\
=-\int_{Q_{\tau}}\left(\vec{g}\left(u_{+}\right) \cdot \nabla u\right) u d x d t+\int_{Q_{\tau}} f_{M}(u) u d x d t .
\end{gathered}
$$


Using the dynamic boundary condition (2.2), we conclude

$$
\begin{gathered}
\int_{Q_{\tau}} u u_{t} d x d t+\int_{Q_{\tau}}\left(\frac{1}{n}+|\nabla u|^{2}\right)^{\frac{p-2}{2}}|\nabla u|^{2} d x d t+\int_{0}^{\tau} \int_{\partial \Omega} \sigma u u_{t} d S d t \\
=-\int_{Q_{\tau}}\left(\vec{g}\left(u_{+}\right) \cdot \nabla u\right) u d x d t+\int_{Q_{\tau}} f_{M}(u) u d x d t .
\end{gathered}
$$

That is,

$$
\begin{aligned}
& \frac{1}{2} \int_{\Omega} u^{2}(x, \tau) d x+\int_{Q_{\tau}}\left(\frac{1}{n}+|\nabla u|^{2}\right)^{\frac{p-2}{2}}|\nabla u|^{2} d x d t+\frac{1}{2} \int_{\partial \Omega} \sigma u^{2}(x, \tau) d S \\
& \quad=\frac{1}{2} \int_{\Omega} u_{0, n}^{2}(x) d x+\frac{1}{2} \int_{\partial \Omega} \sigma u_{0, n}^{2}(x) d S-\int_{Q_{\tau}}\left(\vec{g}\left(u_{+}\right) \cdot \nabla u\right) u d x d t+\int_{Q_{\tau}} f_{M}(u) u d x d t
\end{aligned}
$$

Notice that $u_{0, n} \leq \sup _{\Omega} u_{0}$,

$$
\left|\int_{Q_{\tau}}\left(\vec{g}\left(u_{+}\right) \cdot \nabla u\right) u d x d t\right| \leq \frac{1}{4} \int_{Q_{\tau}}|\nabla u|^{p} d x d t+C \int_{Q_{\tau}}\left(\left|\vec{g}\left(u_{+}\right)\right| u\right)^{\frac{p}{p-1}} d x d t
$$

and

$$
\begin{aligned}
|\nabla u|^{p} & \leq\left(\frac{1}{n}+|\nabla u|^{2}\right)^{\frac{p-2}{2}}|\nabla u|^{2}, \quad p \geq 2, \\
|\nabla u|^{p} & \leq 2^{\frac{2-p}{2}}\left(\frac{1}{n}+|\nabla u|^{2}\right)^{\frac{p-2}{2}}|\nabla u|^{2}+\left(\frac{1}{n}\right)^{\frac{p}{2}} \\
& \leq 2\left(\frac{1}{n}+|\nabla u|^{2}\right)^{\frac{p-2}{2}}|\nabla u|^{2}+1, \quad 1<p<2 .
\end{aligned}
$$

Lemma 2.4 implies $|u| \leq \sup _{\Omega} u_{0}+M T$ for $(x, t) \in Q_{T}$. Therefore,

$$
\int_{\Omega} u^{2}(x, t) d x, \quad \int_{\partial \Omega} \sigma u^{2}(x, t) d S, \quad \int_{Q_{T}}|\nabla u|^{p} d x d t \leq C(M, T)
$$

If $T=\delta_{0}$, Lemma 2.6 shows $|u| \leq \sup _{\Omega} u_{0}+M_{0} \delta_{0}$ for $(x, t) \in Q_{\delta_{0}}$, which is a uniform bound independent of $n, M$.

Lemma 2.8 Suppose that $\sigma$ does not depend on time and $p \geq 2$. For any given $T>0, M>0$, there exists a constant $C=C(M, T)$ independent of $n$ such that

$$
\int_{\Omega}\left|\nabla u_{n, M}\right|^{p} d x, \quad \int_{Q_{T}}\left|\frac{\partial u_{n, M}}{\partial t}\right|^{2} d x d t, \quad \int_{0}^{T} \int_{\Omega} \sigma\left|\frac{\partial u_{n, M}}{\partial t}\right|^{2} d S d t \leq C .
$$

Moreover, if $T=\delta_{0}$ (the constant in Lemma 2.6), then the constant $C=C\left(\delta_{0}\right)$ is independent of $n, M$.

Proof We write $u=u_{n, M}$ in this proof for the sake of convenience. Since $f_{M}, g\left(s_{+}\right)$are Lipschitz continuous, the classical regularity results in [9] imply that $u_{t} \in C^{1,0}(\bar{\Omega} \times(0, T))$. 
Multiplying equation (2.1) by $u_{t}$ and integrating over $Q_{\tau}$, we have

$$
\begin{gathered}
\int_{Q_{\tau}} u_{t}^{2} d x d t+\int_{Q_{\tau}}\left(\frac{1}{n}+|\nabla u|^{2}\right)^{\frac{p-2}{2}} \nabla u \cdot \nabla u_{t} d x d t+\int_{0}^{\tau} \int_{\partial \Omega} \sigma u_{t}^{2} d S d t \\
=-\int_{Q_{\tau}}\left(\vec{g}\left(u_{+}\right) \cdot \nabla u\right) u_{t} d x d t+\int_{Q_{\tau}} f_{M}(u) u_{t} d x d t
\end{gathered}
$$

Next, we show that

$$
\begin{aligned}
\int_{Q_{\tau}} & \left(\frac{1}{n}+|\nabla u|^{2}\right)^{\frac{p-2}{2}} \nabla u \cdot \nabla u_{t} d x d t \\
= & \int_{Q_{\tau}} \frac{1}{2} \frac{\partial}{\partial t} \int_{0}^{|\nabla u(x, t)|^{2}}\left(s+\frac{1}{n}\right)^{\frac{p-2}{2}} d s d x d t \\
= & \int_{Q_{\tau}} \frac{1}{p} \frac{\partial}{\partial t}\left(\left(\frac{1}{n}+|\nabla u(x, t)|^{2}\right)^{\frac{p}{2}}-\left(\frac{1}{n}\right)^{\frac{p}{2}}\right) d x d t \\
= & \frac{1}{p} \int_{\Omega}\left(\frac{1}{n}+|\nabla u(x, \tau)|^{2}\right)^{\frac{p}{2}} d x-\frac{1}{p} \int_{\Omega}\left(\frac{1}{n}+\left|\nabla u_{0, n}(x)\right|^{2}\right)^{\frac{p}{2}} d x \\
\geq & \frac{1}{p} \int_{\Omega}|\nabla u(x, \tau)|^{p} d x-\frac{1}{p} 2^{\frac{p}{2}} \int_{\Omega}\left|\nabla u_{0, n}\right|^{p} d x-\frac{1}{p} 2^{\frac{p}{2}}|\Omega| .
\end{aligned}
$$

Young's inequality yields

$$
\begin{aligned}
&\left|-\int_{Q_{\tau}}\left(\vec{g}\left(u_{+}\right) \cdot \nabla u\right) u_{t} d x d t\right| \leq \frac{1}{4} \int_{Q_{\tau}} u_{t}^{2} d x d t+\int_{Q_{\tau}}\left|\vec{g}\left(u_{+}\right)\right|^{2}|\nabla u|^{2} d x d t \\
& \leq \frac{1}{4} \int_{Q_{\tau}} u_{t}^{2} d x d t+C(M, T) \int_{Q_{\tau}}|\nabla u|^{p} d x d t, \quad p \geq 2, \\
&\left|\int_{Q_{\tau}} f_{M}(u) u_{t} d x d t\right| \leq \frac{1}{4} \int_{Q_{\tau}} u_{t}^{2} d x d t+\int_{Q_{\tau}} f_{M}^{2}(u) d x d t .
\end{aligned}
$$

We conclude the estimate.

Now, we define the following two types of weak solutions of problem (1.1)-(1.3).

Definition 2.1 A function $u \in L^{p}\left((0, T) ; W^{1, p}(\Omega)\right)$ is called a local weak solution of problem (1.1)-(1.3) if the integral equality

$$
\begin{aligned}
& -\int_{\Omega} u_{0} \varphi d x-\int_{Q_{T}} u \varphi_{t} d x d t+\int_{Q_{T}}|\nabla u|^{p-2} \nabla u \cdot \nabla \varphi d x d t-\int_{0}^{T} \int_{\partial \Omega} u(\sigma \varphi)_{t} d S d t \\
& =-\int_{Q_{T}}(\vec{g}(u) \cdot \nabla u) \varphi d x d t+\int_{Q_{T}} f(u) \varphi d x d t
\end{aligned}
$$

holds for any $\varphi \in C^{\infty}\left(\bar{Q}_{T}\right)$ that satisfies $\varphi(x, T)=0$ for $x \in \bar{\Omega}, \varphi(x, 0)=0$ for $x \in \partial \Omega$.

Definition 2.2 A function $u \in L^{p}\left((0, T) ; W^{1, p}(\Omega)\right)$ is called a local strong solution of problem (1.1)-(1.3) if $u_{t} \in L^{2}\left(Q_{T}\right), u$ is the a.e. limit function of a subsequence $\left\{u_{n_{k}, M_{k}}\right\}$ of classical solutions to the regularized problem (2.1)-(2.3), and the integral equality (2.7) holds for any $\varphi \in C^{\infty}\left(\bar{Q}_{T}\right)$ that satisfies $\varphi(x, T)=0$ for $x \in \bar{\Omega}, \varphi(x, 0)=0$ for $x \in \partial \Omega$. 
Theorem 2.1 Suppose that $\sigma$ does not depend on time. Problem (1.1)-(1.3) admits at least one local weak solution.

Proof For any $T>0, \varphi \in C^{\infty}\left(\bar{Q}_{T}\right)$ that satisfies $\varphi(x, T)=0$ for $x \in \bar{\Omega}, \varphi(x, 0)=0$ for $x \in \partial \Omega$, multiplying (2.1) by $\varphi$, integrating over $Q_{T}$, we have

$$
\begin{aligned}
\int_{Q_{T}} & \frac{\partial u_{n, M}}{\partial t} \varphi d x d t+\int_{Q_{T}}\left(\frac{1}{n}+\left|\nabla u_{n, M}\right|^{2}\right)^{\frac{p-2}{2}} \nabla u_{n, M} \cdot \nabla \varphi d x d t \\
& -\int_{0}^{T} \int_{\partial \Omega}\left(\frac{1}{n}+\left|\nabla u_{n, M}\right|^{2}\right)^{\frac{p-2}{2}} \nabla u_{n, M} \cdot v \varphi d S d t \\
= & -\int_{Q_{T}}\left(\vec{g}\left(\left(u_{n, M}\right)_{+}\right) \cdot \nabla u_{n, M}\right) \varphi d x d t+\int_{Q_{T}} f_{M}\left(u_{n, M}\right) \varphi d x d t .
\end{aligned}
$$

By the dynamic boundary condition (2.2), we obtain

$$
\begin{aligned}
& -\int_{0}^{T} \int_{\partial \Omega}\left(\frac{1}{n}+\left|\nabla u_{n, M}\right|^{2}\right)^{\frac{p-2}{2}} \nabla u_{n, M} \cdot v \varphi d S d t \\
& =\int_{0}^{T} \int_{\partial \Omega} \sigma \frac{\partial u_{n, M}}{\partial t} \varphi d S d t=-\int_{0}^{T} \int_{\partial \Omega} u_{n, M}(\sigma \varphi)_{t} d S d t .
\end{aligned}
$$

Thus,

$$
\begin{aligned}
& -\int_{\Omega} u_{0, n} \varphi d x-\int_{Q_{T}} u_{n, M} \varphi_{t} d x d t+\int_{Q_{T}}\left(\frac{1}{n}+\left|\nabla u_{n, M}\right|^{2}\right)^{\frac{p-2}{2}} \nabla u_{n, M} \cdot \nabla \varphi d x d t \\
& \quad=\int_{0}^{T} \int_{\partial \Omega} u_{n, M}(\sigma \varphi)_{t} d S d t-\int_{Q_{T}}\left(\vec{g}\left(u_{n, M}\right) \cdot \nabla u_{n, M}\right) \varphi d x d t+\int_{Q_{T}} f_{M}\left(u_{n, M}\right) \varphi d x d t .
\end{aligned}
$$

By the uniform estimates in Lemma 2.6 and Lemma 2.7, there exist a subsequence $\left\{u_{n_{k}, M_{k}}\right\}$ $\left(n_{k} \rightarrow \infty, M_{k} \rightarrow \infty\right.$, as $\left.k \rightarrow \infty\right)$ and a function $u \in L^{p}\left(\left(0, \delta_{0}\right) ; W^{1, p}(\Omega)\right)$ such that $u_{n_{k}, M_{k}}$ converges weakly to $u$ in $L^{2}\left(Q_{\delta_{0}}\right), \nabla u_{n_{k}, M_{k}}$ converges weakly to $\nabla u$ in $L^{p}\left(Q_{\delta_{0}}\right)$, and $u_{n_{k}, M_{k}}$ converges weakly to $u$ in $L^{p}\left(\partial \Omega \times\left(0, \delta_{0}\right)\right)$ in the sense of trace. Hence the above integral equality converges to (2.7) for $T=\delta_{0}$ and $u$ is a local weak solution to problem (1.1)(1.3).

Theorem 2.2 Suppose that $\sigma$ does not depend on time and $p \geq 2$. Problem (1.1)-(1.3) admits at least one local strong solution.

Proof By the uniform estimates in Lemma 2.6, Lemma 2.7, and Lemma 2.8, the norms $\left\|u_{n, M}\right\|_{H^{1}\left(Q_{\delta_{0}}\right)},\left\|\nabla u_{n, M}\right\|_{L^{p}\left(Q_{\delta_{0}}\right)}$ are uniformly bounded. There exist a subsequence $\left\{u_{n_{k}, M_{k}}\right\}$ $\left(n_{k} \rightarrow \infty, M_{k} \rightarrow \infty\right.$, as $\left.k \rightarrow \infty\right)$ and a function $u \in L^{p}\left(\left(0, \delta_{0}\right) ; W^{1, p}(\Omega)\right), u_{t} \in L^{2}\left(Q_{\delta_{0}}\right)$ such that $\left\{u_{n_{k}, M_{k}}\right\}$ converges weakly to $u$ in $H^{1}\left(Q_{\delta_{0}}\right), \nabla u_{n_{k}, M_{k}}$ converges weakly to $\nabla u$ in $L^{p}\left(Q_{\delta_{0}}\right)$. Hence $u_{n_{k}, M_{k}} \rightarrow u$ almost everywhere and the integral equality (2.7) holds.

Remark For any given $M>0$, by the estimates in Lemma 2.7 and Lemma 2.8, using the diagonal procedure, we can choose a subsequence $\left\{u_{n_{k}, M}\right\}$ ( $\left\{n_{k}\right\}$ might depend on $M$ ) and a function $u_{M}$ such that $u_{n_{k}, M}$ converges to $u_{M}$ on $Q_{T}$ for any $T>0$ in the manner stated in the proof of Theorem 2.2. Furthermore, we can verify that $u_{M}$ is the global strong solution 
to the following equation:

$$
\frac{\partial u}{\partial t}=\operatorname{div}\left(|\nabla u|^{p-2} \nabla u\right)-\vec{g}(u) \cdot \nabla u+f_{M}(u)
$$

coupled with the initial-boundary value conditions (1.2)-(1.3). Using the diagonal procedure again, we can choose a subsequence $\left\{n_{k}\right\}$ independent of $M$ and then choose $u_{M}$ such that $u_{n_{k}, M}$ converges to $u_{M}$ for any $M \in \mathbb{Z}^{+}$in the same manner. Lemma 2.5 implies $u_{n_{k}, M_{1}} \geq u_{n_{k}, M_{2}}$ for $M_{1} \geq M_{2}$. Thus, $\left\{u_{M}\right\}_{M \in \mathbb{Z}^{+}}$is monotone with respect to $M$. Define

$$
T^{*}=\sup \left\{T>0 ; \sup _{M \in \mathbb{Z}^{+}} \sup _{(x, t) \in \bar{\Omega} \times(0, T)} u_{M}(x, t)<\infty\right\},
$$

and

$$
u(x, t)=\sup _{M \in \mathbb{Z}^{+}} u_{M}(x, t), \quad(x, t) \in \bar{\Omega} \times\left(0, T^{*}\right) .
$$

Lemma 2.6 shows $T^{*} \geq \delta_{0}$. Similar to the proof of Theorem 2.1 and Theorem 2.2, we can prove that $u$ is a strong solution to problem (1.1)-(1.3) with maximal existence time $T^{*}$.

\section{Global existence}

In this section, we study the global existence of local strong solutions to problem (1.1)(1.3) defined in Section 2. We need to find an appropriate upper-solution to the regularized problem (2.1)-(2.3) which is independent of $n, M$ and exists globally. If $p=2$, the $p$-Laplacian is reduced to Laplacian, so we only consider $p>2$ in this section.

Lemma 3.1 Let $\alpha=\frac{p-1}{p-2}, p>2, K>0, \eta \in C^{1}([0,+\infty))$. For a fixed integer $1 \leq j \leq N$, define $\underline{x}_{j}=\min _{\bar{\Omega}} x_{j}, \bar{x}_{j}=\max _{\bar{\Omega}} x_{j}$, and

$$
U(x, t)=\frac{1}{\alpha}\left(K e^{\eta(t)}+x_{j}-\bar{x}_{j}\right)^{\alpha}, \quad x \in \bar{\Omega}, t \geq 0 .
$$

Then $U$ is an upper solution of the regularized problem (2.1)-(2.3) provided

$$
\begin{aligned}
& K e^{\eta(0)}+\underline{x}_{j}-\bar{x}_{j} \geq 1, \quad \frac{1}{\alpha}\left(K e^{\eta(0)}+\underline{x}_{j}-\bar{x}_{j}\right)^{\alpha} \geq \sup _{\Omega} u_{0}, \\
& \eta^{\prime}(t) \geq \alpha 2^{p}, \quad \sigma(x, t) \eta^{\prime}(t) \geq 2^{p}, \quad x \in \partial \Omega, t \geq 0,
\end{aligned}
$$

and

$$
g_{j}(s) s^{\frac{1}{p-1}} \geq f(s), \quad s \geq \frac{1}{\alpha}\left(K e^{\eta(0)}+\underline{x}_{j}-\bar{x}_{j}\right)^{\alpha} .
$$

Proof By a simple computation, we have

$$
\begin{aligned}
& \operatorname{div}\left(\left(\frac{1}{n}+|\nabla u|^{2}\right)^{\frac{p-2}{2}} \nabla u\right) \\
& \quad=\left(\frac{1}{n}+|\nabla u|^{2}\right)^{\frac{p-2}{2}} \Delta u+(p-2)\left(\frac{1}{n}+|\nabla u|^{2}\right)^{\frac{p-2}{2}-1} \frac{\partial u}{\partial x_{i}} \frac{\partial u}{\partial x_{j}} \frac{\partial^{2} u}{\partial x_{i} \partial x_{j}} .
\end{aligned}
$$


Notice that $\alpha>1$ and $(\alpha-1)(p-1)=\alpha$. We show that

$$
\begin{aligned}
& |\nabla U|=\frac{\partial U}{\partial x_{j}}=\left(K e^{\eta(t)}+x_{j}-\bar{x}_{j}\right)^{\alpha-1} \geq 1, \\
& U_{t}=\left(K e^{\eta(t)}+x_{j}-\bar{x}_{j}\right)^{\alpha-1} K e^{\eta(t)} \eta^{\prime}(t) \geq\left(K e^{\eta(t)}+x_{j}-\bar{x}_{j}\right)^{\alpha} \eta^{\prime}(t), \\
& \left|\left(\frac{1}{n}+|\nabla U|^{2}\right)^{\frac{p-2}{2}} \nabla U \cdot v\right| \leq 2^{\frac{p-2}{2}}|\nabla U|^{p-1} \leq 2^{p}\left(K e^{\eta(t)}+x_{j}-\bar{x}_{j}\right)^{\alpha}, \\
& \operatorname{div}\left(\left(\frac{1}{n}+|\nabla U|^{2}\right)^{\frac{p-2}{2}} \nabla U\right) \leq 2^{\frac{p-2}{2}}|\nabla U|^{p-2} \Delta U+(p-2) \max \left\{2^{\frac{p-2}{2}-1}, 1\right\}|\nabla U|^{p-2} \frac{\partial^{2} U}{\partial x_{j}^{2}} \\
& \leq \alpha 2^{p}\left(K e^{\eta(t)}+x_{j}-\bar{x}_{j}\right)^{\alpha-1} .
\end{aligned}
$$

Thus,

$$
\begin{aligned}
B_{n}[U]=\sigma U_{t} & -\left(\frac{1}{n}+|\nabla U|^{2}\right)^{\frac{p-2}{2}} \nabla U \cdot v \geq 0, \\
U_{t}-F_{n, M}[U] & =U_{t}-\operatorname{div}\left(\left(\frac{1}{n}+|\nabla U|^{2}\right)^{\frac{p-2}{2}} \nabla U\right)+\vec{g}(U) \cdot \nabla U-f(U) \\
& \geq g_{j}(U)(\alpha U)^{\frac{1}{p-1}}-f(U) \geq 0, \quad x \in \Omega, t>0,
\end{aligned}
$$

and

$$
U(x, 0)=\frac{1}{\alpha}\left(K e^{\eta(0)}+x_{j}-\bar{x}_{j}\right)^{\alpha} \geq \sup _{\Omega} u_{0} \geq u_{0, n}(x), \quad x \in \bar{\Omega} .
$$

Lemma 2.2 implies that $U(x, t)$ is an upper solution of problem (2.1)-(2.3).

Now we give some conditions on the functions $f, g$, and $\sigma$, which ensure the global existence of local solutions.

Theorem 3.1 Suppose $p>2,\left(\inf _{x \in \partial \Omega} \sigma(x, \cdot)\right)^{-1} \in L_{\text {loc }}^{1}([0,+\infty))$, there exist an integer $1 \leq$ $j \leq N$ and a constant $M>1$ such that

$$
g_{j}(s) s^{\frac{1}{p-1}} \geq f(s), \quad s \geq M .
$$

Then the strong solution of problem (1.1)-(1.3) is a global solution.

Proof Take $K=\max \left\{1,\left(\alpha \sup _{\Omega} u_{0}\right)^{\frac{1}{\alpha}},(\alpha M)^{\frac{1}{\alpha}}\right\}+\bar{x}_{j}-\underline{x}_{j}$, and define

$$
\eta(t)=2^{p} \int_{0}^{t}\left(\inf _{x \in \partial \Omega} \sigma(x, \tau)\right)^{-1} d \tau+\alpha 2^{p} t
$$

where $\alpha, \bar{x}_{j}, \underline{x}_{j}$ are the constants defined in Lemma 3.1. Thus, $U(x, t)=\frac{1}{\alpha}\left(K e^{\eta(t)}+x_{j}-\bar{x}_{j}\right)^{\alpha}$ is an upper solution to the regularized problem (2.1)-(2.3) for any $n \in \mathbb{Z}^{+}$and $M>0$. That 
is, $u_{n, M}(x, t) \leq U(x, t)$ for $x \in \bar{\Omega}$ and $t \geq 0$. According to the definition of strong solution, we have $u(x, t) \leq U(x, t)$. Hence $u$ does not blow up in finite time.

\section{Blow-up}

In this section, we investigate the blow-up phenomenon of problem (1.1)-(1.3). We need to construct a family of lower solutions of the regularized problem (2.1)-(2.3) whose supremum blows up in finite time.

Lemma 4.1 Suppose that $p>2, \Omega$ is a convex domain, and there exist constants $C_{1}, C_{2}>0$ such that

$$
f(s) \geq C_{1} s^{p-1}, \quad|\vec{g}(s)| \leq C_{2} s^{p-2}, \quad s \geq 0
$$

Choose $x_{0} \in \Omega$ with $B_{r}\left(x_{0}\right) \subset \Omega, r>0$. For $A, B>0$ and $\varphi_{M} \in C^{1}([0,+\infty))$, define

$$
\nu_{M}(x, t)=\left(A-B\left|x-x_{0}\right|^{2}\right) \varphi_{M}(t), \quad x \in \bar{\Omega}, t \geq 0 .
$$

Then the function $v_{M}$ is a lower solution of the regularized problem (2.1)-(2.3) provided

$$
\begin{aligned}
& A \geq 2 B d^{2}, \quad 2 B d \varphi_{M}(0) \geq 1, \quad A \varphi_{M}(0) \leq \inf _{\Omega} u_{0}, \quad C_{1}\left(A \varphi_{M}(t)\right)^{p-1} \leq M, \\
& \varphi_{M}^{\prime} \geq 0, \quad \sigma A \varphi_{M}^{\prime} \leq(2 B r)^{p-1} \delta \varphi_{M}^{p-1}, \quad A \varphi_{M}^{\prime} \leq K \varphi_{M}^{p-1},
\end{aligned}
$$

where $d=\sup _{\Omega}\left|x-x_{0}\right|, \delta=\inf _{\partial \Omega} \frac{x-x_{0}}{\left|x-x_{0}\right|} \cdot v>0$ (by the convexity of $\Omega$ ), and

$$
K=\frac{1}{2} C_{1}\left(\frac{1}{2} A\right)^{p-1}-2^{p}(2 B)^{p-1} d^{p-2}(N+p)-\left(\frac{1}{2} C_{1}\right)^{-(p-2)} C_{2}^{p-1}(2 B d)^{p-1}
$$

Proof Let $\rho(x)=\left|x-x_{0}\right|$. A direct calculation shows

$$
\begin{aligned}
\nabla v_{M}=-2 B \varphi_{M}\left(x-x_{0}\right), \quad \frac{\partial v_{M}}{\partial t} & =\left(A-B \rho^{2}\right) \varphi_{M}^{\prime}(t) \\
\left(\frac{1}{n}+\left|\nabla v_{M}\right|^{2}\right)^{\frac{p-2}{2}} \nabla v_{M}=-\left(\frac{1}{n}+(2 B)^{2} \rho^{2} \varphi_{M}^{2}\right)^{\frac{p-2}{2}} 2 B \varphi_{M}\left(x-x_{0}\right) & \\
\operatorname{div}\left(\left(\frac{1}{n}+\left|\nabla v_{M}\right|^{2}\right)^{\frac{p-2}{2}} \nabla v_{M}\right)= & -\left(\frac{1}{n}+(2 B)^{2} \rho^{2} \varphi_{M}^{2}\right)^{\frac{p-2}{2}} 2 N B \varphi_{M} \\
& -(p-2)\left(\frac{1}{n}+(2 B)^{2} \rho^{2} \varphi_{M}^{2}\right)^{\frac{p-2}{2}-1}(2 B)^{2} \rho^{2} \varphi_{M}^{2} 2 B \varphi_{M} \\
= & -\left(\frac{1}{n}+(2 B)^{2} \rho^{2} \varphi_{M}^{2}\right)^{\frac{p-2}{2}-1} 2 B \varphi_{M} \\
& \cdot\left(\left(\frac{1}{n}+(2 B)^{2} \rho^{2} \varphi_{M}^{2}\right) N+(p-2)(2 B)^{2} \rho^{2} \varphi_{M}^{2}\right) .
\end{aligned}
$$


Thus, we have

$$
\begin{aligned}
\left|\operatorname{div}\left(\left(\frac{1}{n}+\left|\nabla v_{M}\right|^{2}\right)^{\frac{p-2}{2}} \nabla v_{M}\right)\right| & \leq\left(\frac{1}{n}+(2 B)^{2} \rho^{2} \varphi_{M}^{2}\right)^{\frac{p-2}{2}}(N+p-1) 2 B \varphi_{M} \\
& \leq\left(1+(2 B d)^{2} \varphi_{M}^{2}\right)^{\frac{p-2}{2}}(N+p-1) 2 B \varphi_{M} \\
& \leq 2^{p}(2 B)^{p-1} d^{p-2}(N+p) \varphi_{M}^{p-1}, \quad x \in \bar{\Omega}, t \leq 0,
\end{aligned}
$$

and

$$
\begin{aligned}
\left(\frac{1}{n}+\left|\nabla v_{M}\right|^{2}\right)^{\frac{p-2}{2}} \nabla v_{M} \cdot v & =-\left(\frac{1}{n}+(2 B)^{2} \rho^{2} \varphi_{M}^{2}\right)^{\frac{p-2}{2}} 2 B \varphi_{M} \rho \frac{x-x_{0}}{\left|x-x_{0}\right|} \cdot v \\
& \leq-\left(\frac{1}{n}+(2 B)^{2} \rho^{2} \varphi_{M}^{2}\right)^{\frac{p-2}{2}} 2 B \varphi_{M} \rho \delta \\
& \leq-(2 B r)^{p-1} \delta \varphi_{M}^{p-1}, \quad x \in \partial \Omega, t \geq 0 .
\end{aligned}
$$

Young's inequality shows

$$
\left|\vec{g}\left(v_{M}\right) \cdot \nabla v_{M}\right| \leq C_{2} v_{M}^{p-2}\left|\nabla v_{M}\right| \leq \frac{1}{2} C_{1} v_{M}^{p-1}+\left(\frac{1}{2} C_{1}\right)^{-(p-2)} C_{2}^{p-1}\left|\nabla v_{M}\right|^{p-1}
$$

We obtain

$$
f_{M}\left(v_{M}\right)=\min \left\{M, f\left(v_{M}\right)\right\} \geq C_{1} v_{M}^{p-1}
$$

and

$$
\begin{aligned}
f_{M}\left(v_{M}\right)-\vec{g} \cdot \nabla v_{M} & \geq \frac{1}{2} C_{1} v_{M}^{p-1}-\left(\frac{1}{2} C_{1}\right)^{-(p-2)} C_{2}^{p-1}\left|\nabla v_{M}\right|^{p-1} \\
& \geq \frac{1}{2} C_{1}\left(\frac{1}{2} A \varphi_{M}\right)^{p-1}-\left(\frac{1}{2} C_{1}\right)^{-(p-2)} C_{2}^{p-1}\left(2 B d \varphi_{M}\right)^{p-1}
\end{aligned}
$$

Furthermore,

$$
\begin{gathered}
\frac{\partial v_{M}}{\partial t}-F_{n, M}\left[v_{M}\right] \leq A \varphi_{M}^{\prime}-K \varphi_{M}^{p-1} \leq 0, \quad(x, t) \in \partial \Omega \times \mathbb{R}^{+}, \\
B_{n}\left[v_{M}\right]=\sigma \frac{\partial v_{M}}{\partial t}+\left(\frac{1}{n}+\left|\nabla v_{M}\right|^{2}\right)^{\frac{p-2}{2}} \frac{\partial v_{M}}{\partial \nu} \\
\leq \sigma A \varphi_{M}^{\prime}-(2 B r)^{p-1} \delta \varphi_{M}^{p-1} \leq 0, \quad(x, t) \in \partial \Omega \times \mathbb{R}^{+}, \\
v_{M}(x, 0) \leq A \varphi_{M}(0) \leq \inf _{\Omega} u_{0} \leq u_{0, n}(x), \quad x \in \bar{\Omega} .
\end{gathered}
$$

Lemma 2.2 implies that $v_{M}$ is a lower solution of problem (2.1)-(2.3).

Theorem 4.1 Suppose that $p>2, \Omega$ is a convex domain, $\sigma \in L^{\infty}\left(\partial \Omega \times \mathbb{R}^{+}\right)$, and there exist constants $C_{1}, C_{2}>0$ such that

$$
f(s) \geq C_{1} s^{p-1}, \quad|\vec{g}(s)| \leq C_{2} s^{p-2}, \quad s \geq 0 .
$$


Then the strong solution of problem (1.1)-(1.3) blows up in finite time provided that $\inf _{\Omega} u_{0}$ is sufficiently large.

Proof Let $x_{0}, r, d, \delta, K$ be as defined in Lemma 4.1. Since $K=K(A, B)$ converges to $K(A, 0)=\frac{1}{2} C_{1}\left(\frac{1}{2} A\right)^{p-1}>0$ as $B$ tends to $0^{+}$, we can choose $A, B>0$ such that

$$
C_{1} A^{p-1}=1, \quad 2 B d^{2} \leq A, \quad K=K(A, B)>0 .
$$

Then set

$$
\varphi_{M}(0)=\frac{1}{2 B d}>0, \quad \bar{\sigma}=\sup _{\partial \Omega \times \mathbb{R}^{+}} \sigma, \quad \gamma=\min \left\{\frac{K}{A}, \frac{(2 B r)^{p-1} \delta}{\bar{\sigma} A}\right\} .
$$

By Lemma 4.1, the function $v_{M}=\left(A-B\left|x-x_{0}\right|^{2}\right) \varphi_{M}(t)$ is a lower solution provided

$$
\inf _{\Omega} u_{0} \geq \frac{A}{2 B d}, \quad \varphi_{M}(t) \leq M^{\frac{1}{p-1}}, \quad 0 \leq \varphi_{M}^{\prime}(t) \leq \gamma \varphi_{M}^{p-1}(t) .
$$

Define

$$
\varphi_{M}(t)=\min \left\{\left(\varphi_{M}^{2-p}(0)-(p-2) \gamma t\right)_{+}^{-\frac{1}{p-2}}, M^{\frac{1}{p-1}}\right\}, \quad t \geq 0 .
$$

Although $\varphi_{M}(t)$ is not $C^{1}$ continuous, we can change the partial derivative $\frac{\partial}{\partial t}$ to the leftward partial derivative $\frac{\partial}{\partial t^{-}}$in the proof of Lemma 2.1, Lemma 2.2, and Lemma 4.1, then we conclude that $v_{M}$ is a lower solution of problem (2.1)-(2.3). Hence $u_{n, M}(x, t) \geq v_{M}(x, t)$ for $(x, t) \in \bar{\Omega} \times \mathbb{R}^{+}$. By the definition of strong solution, we have

$$
u(x, t) \geq \sup _{M \in \mathbb{Z}^{+}} v_{M}(x, t), \quad x \in \bar{\Omega}, t \in\left(0, T_{0}\right),
$$

where $T_{0}=\frac{(2 B d)^{p-2}}{(p-2) \gamma}$. Since $v_{M}$ blows up at finite time $T_{0}$, the strong solution $u$ must blow up at time $T^{*} \leq T_{0}$.

\section{Competing interests}

The authors declare that they have no competing interests.

\section{Authors' contributions}

The authors declare that the study was realized in collaboration with the same responsibility. All authors read and approved the final manuscript.

\section{Acknowledgements}

The first author was supported in part by the Scientific Research Foundation of Graduate School of South China Normal University (No. 2013kyjj022). The second author and the third author were supported in part by NNSFC (No. 11071099). The third author was supported in part by CPSF (No. 2015M572301) and the Fundamental Research Funds for the Central Universities.

Received: 27 August 2015 Accepted: 9 October 2015 Published online: 24 October 2015

\section{References}

1. Escher, J: Quasilinear parabolic systems with dynamical boundary conditions. Commun. Partial Differ. Equ. 18, 1309-1364 (1993)

2. Mailly-Pincet, G, Rault, J-F: Nonlinear convection in reaction-diffusion equations under dynamical boundary conditions. Electron. J. Differ. Equ. 2013, 10 (2013)

3. von Below, J, Mailly-Pincet, G: Blow up for reaction diffusion equation under dynamical boundary conditions. Commun. Partial Differ. Equ. 28, 223-247 (2003) 
4. Gal, CG, Warma, M: Well posedness and the global attractor of some quasilinear parabolic equations with nonlinear dynamic boundary conditions. Differ. Integral Equ. 23, 327-358 (2010)

5. Andreu, F, Mazón, JM, Toledo, J: A degenerate elliptic-parabolic problem with nonlinear dynamical boundary conditions. Interfaces Free Bound. 8, 447-479 (2006)

6. von Below, J, Mailly-Pincet, G: Blow up for some nonlinear parabolic problems with convection under dynamical boundary conditions. In: Discrete and Continuous Dynamical Systems, Dynamical Systems and Differential Equations, Proceedings of the 6th AIMS International Conference, Supplement, pp. 1031-1041 (2007)

7. Constantin, A, Escher, J: Global existence for fully parabolic boundary value problems. Nonlinear Differ. Equ. Appl. 13, 91-118 (2006)

8. von Below, J, De Coster, C: A qualitative theory for parabolic problems under dynamical boundary conditions. J. Inequal. Appl. 5, 467-486 (2000)

9. Ladyženskaya, OA, Solonnikov, VA, Uraltseva, NN: Linear and Quasilinear Equations of Parabolic Type. Transl. of Math. Monogr., vol. 23. Am. Math. Soc. , Providence (1968)

Submit your manuscript to a SpringerOpen ${ }^{\circ}$ journal and benefit from:

- Convenient online submission

Rigorous peer review

- Immediate publication on acceptance

- Open access: articles freely available online

- High visibility within the field

- Retaining the copyright to your article 